\title{
Time-resolved pump-probe experiments beyond the jitter limitations at FLASH
}

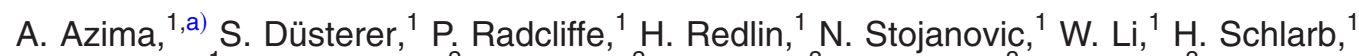 \\ J. Feldhaus, ${ }^{1}$ D. Cubaynes, ${ }^{2}$ M. Meyer, ${ }^{2}$ J. Dardis, ${ }^{3}$ P. Hayden, ${ }^{3}$ P. Hough, ${ }^{3}$ \\ V Richardson, ${ }^{3}$ E. T. Kennedy, ${ }^{3}$ and J. T. Costello ${ }^{3}$ \\ ${ }^{1}$ DESY, Notkestr. 85, D-22607 Hamburg, Germany \\ ${ }^{2}$ LIXAM/CNRS, UMR 8624, Centre Universitaire Paris-Sud, Bâtiment 350, F-91405 Orsay Cedex, France \\ ${ }^{3}$ National Center for Plasma Science and Technology and School of Physical Sciences, Dublin City \\ University, Dublin 9, Ireland
}

(Received 9 January 2009; accepted 11 March 2009; published online 7 April 2009)

\begin{abstract}
Using a noninvasive, electro-optically based electron bunch arrival time measurement at FLASH (free electron laser in Hamburg) the temporal resolution of two-color pump-probe experiments has been significantly improved. The system determines the relative arrival time of the extended ultraviolet pulse of FLASH and an amplified Ti:sapphire femtosecond-laser pulse at the interaction region better than $90 \mathrm{fs}$ rms. In a benchmarking pump-probe experiment using two-color above threshold ionization of noble gases, an enhancement in the timing resolution by a factor of 4 compared to the uncorrected data is obtained. (c) 2009 American Institute of Physics.
\end{abstract}

[DOI: $10.1063 / 1.3111789$ ]

Progress in generation and application of ultrashort laser pulses over the past two decades has been tremendous. Efforts toward decreasing the pulse duration and the concomitant widening of the spectral range are steadily increasing, thereby enabling the investigation of dynamical phenomena on faster and faster timescales. At FLASH (free electron laser in Hamburg), ${ }^{1}$ in particular, investigations on the phenomena in the extended ultraviolet (XUV) regime with pulses of only few tens of femtoseconds are now possible, with a worldwide unrivalled high power such that a whole new class of experiments is accessible. The pump-probe excitation scheme is the most promising concept to study dynamical processes on the femtosecond level. Two ultrashort pulses are required for this kind of experiment, first, initiating a reaction and the second permitting the observation of the induced changes. The temporal resolution of this scheme is determined by precise knowledge of the delay between both pulses and, ultimately, by their duration. At FLASH the XUV-free electron laser (XUV-FEL) pulse can be combined with an optical laser pulse. This scheme has already been utilized from the beginning of its operation as a user facility in 2005 and has led to several exciting results, e.g., ${ }^{2-6}$ two complementary laser systems are available. First, a system delivering a high energy pulse $[>10 \mathrm{~mJ}, 120 \mathrm{fs}$ full width at half maximum (FWHM)], albeit the repetition rate is limited to 10 pulses per second. The second system provides trains of $20 \mu \mathrm{J}$ pulses (120 fs FWHM) that map exactly onto the complex timing pattern of the FEL, delivering up to 4000 pulses per second. Since the FEL and the optical laser are independent sources of femtosecond pulses the synchronization between both is of vital importance to perform well defined pump-probe experiments. However, due to technical limitations the synchronization is compromised by the inherent timing jitter reducing the effective temporal resolution from the pulse duration limit of $120 \mathrm{fs}(\mathrm{FWHM} \simeq 50 \mathrm{fs})$ root

\footnotetext{
${ }^{\text {a) }}$ Author to whom correspondence should be addressed. Electronic mail: armin.azima@desy.de.
}

mean square (rms) width (since the FEL pulse has a pulse duration of only few tens of femtoseconds) to about $250 \mathrm{fs}$ rms. This jitter is dominated by the accelerator itself. Our approach to increase the temporal resolution is to measure the arrival time of the pulses during a pump-probe experiment independently and shot by shot without disturbing the experiment. After the experiment the information about the "real" arrival time is accessible to the investigators and can be used to assign the correct arrival time of each data point. An important prerequisite of the scheme is the shot-by-shot data acquisition in the pump-probe experiment. Averaged data cannot be corrected afterwards. In this way the influence of the jitter on the temporal resolution is compensated, leaving only the detection error on the arrival time measurement.

The FLASH generates ultrashort XUV pulses of unprecedented intensity (several gigawatts) in the spectral range from 7 to $50 \mathrm{~nm}$ with pulse durations of $<30 \mathrm{fs}$. The optical pulses are generated by an amplified Ti:sapphire oscillator operating at a wavelength of $800 \mathrm{~nm}$ in the near-infrared (NIR) range and amplified up to $20 \mu \mathrm{J}$ in an optical parametric amplifier. The pulse duration at the experimental site is $120 \mathrm{fs}$ FWHM. The pulse pattern of both sources consists of pulse trains with up to 800 pulses having a repetition rate of $1 \mathrm{MHz}$ within the burst and a burst repetition rate of $5 \mathrm{~Hz}$. The setup to determine the relative arrival time between both sources at FLASH is named "timing by electro-optical sampling" abbreviated as TEO. Since the direct determination of the arrival time difference between the FEL-XUV pulse and the NIR pulse presents several difficulties and is not offhand parasitically installable without disturbing the actual pumpprobe experiment, ${ }^{7}$ the only feasible option was to measure the arrival time between the accelerated electrons and the NIR laser pulses (Fig. 1). The TEO diagnostic determines the precise relative arrival time $T_{e^{-}}$of the electron bunch and the NIR laser pulse in the accelerator tunnel right in front of the self-amplified spontaneous emission emitting undulator section and it does so in a fully parasitic way. The diagnostic utilizes the Pockels effect in combination with a spatial en- 


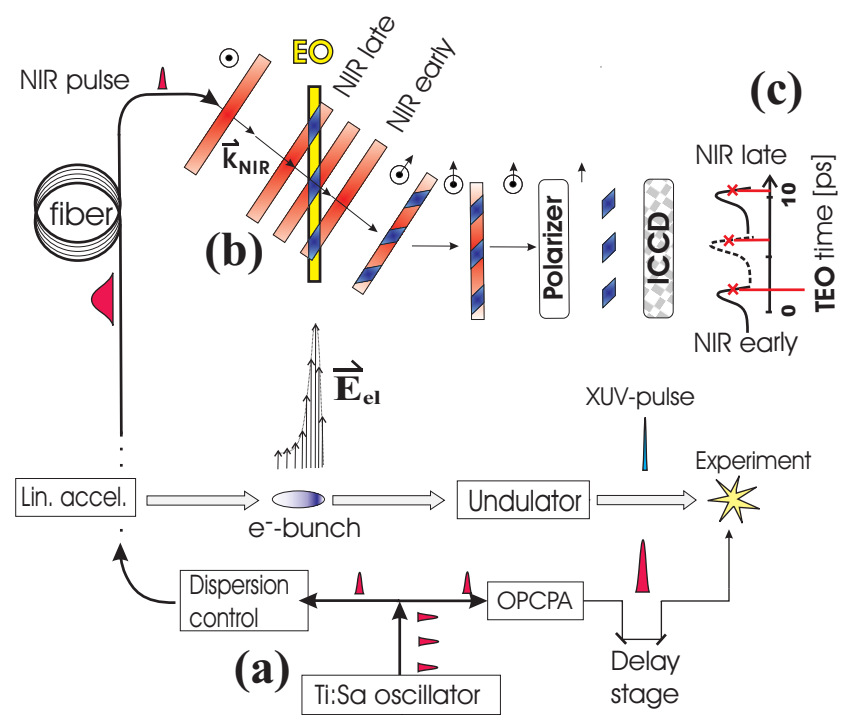

FIG. 1. (Color online) Overview of the TEO setup at FLASH. The relative arrival time between electron bunch and NIR pulse is measured inside the accelerator tunnel. (a) The short NIR pulses of a Ti:sapphire oscillator are transported via a single-mode, polarization maintaining glass fiber. (b) Due to a $45^{\circ}$ angle of incidence, the transversal position of the polarization modulation inside the optical beam profile depends on the relative arrival time of the NIR pulse and the electron bunch at the EO crystal. When the laser pulse arrives later than the electron bunch, the modulation moves within the transversal beam profile to the left (looking along beam direction) (c) The signal detection is performed in a crossed polarizer setup. The arrival time of the electron bunch is measured shot-by-shot.

coding technique to map time onto space ${ }^{8}$ and determine the arrival time of each electron bunch on a shot-by-shot basis.

The NIR laser system is situated in a laser laboratory at the end of the FEL linear accelerator in close proximity to the pump-probe experiments. For the TEO measurement, the pulses generated by the Ti:sapphire oscillator running with 108.3 MHz repetition rate are used directly without amplification. These pulses are transported to the TEO setup in the accelerator tunnel by a $153 \mathrm{~m}$ long single-mode, polarization maintaining glass fiber (Fig. 1). The laser pulse transport system is a key feature of the TEO experiment. The dispersion of the fiber has to be compensated for, so that in the TEO crystal, a nearly Fourier limited pulse is available. Additionally, the fiber length is modulated by thermal expansion and microphonics, which cause slow drifts and fast jitter of about $60-100$ fs rms and $20-30 \mathrm{fs}$ rms on a time scale of minutes and seconds, respectively. ${ }^{9}$ These changes can be compensated by an active path length stabilization. ${ }^{10}$ Abovethreshold ionization is a way to obtain information about the temporal overlap of femtosecond pulses from the XUV-FEL and the NIR laser. ${ }^{3,4}$ The XUV beam is focused inside a low pressure Xe gas target at $10^{-6}$ mbar to ionize the gas atoms. An amplified NIR laser pulse and a collinear XUV-FEL pulse are focused into the gas. Once the two laser pulses overlap in space and time, the electrical field of the NIR laser pulse modulates the kinetic energy of the photoelectrons by $N \times \hbar \omega_{\text {IR }}= \pm 1.55 \mathrm{eV} \times N$, where $N$ defines the order of the detected sideband. Hence, photoelectron spectra show additional sidebands shifted by multiples of the NIR photon energy from the main photoelectron peak. The strength of the sidebands and the visibility of higher order sidebands depend on the degree of overlap of both pulses (XUV and NIR). Experience shows that spatial overlap is rather stable once it is well aligned. Thus, the sideband strength is a very sensitive indicator for the temporal overlap of both pulses.

Two series of experiments on Xe atoms were carried out at FLASH with the experimental setup described in detail in Ref. 4. In the first experiment the delay line in the path of the NIR laser was fixed at a position for optimal temporal overlap between both laser pulses and a set of 2000 spectra was recorded, where only the temporal jitter between FEL and NIR laser caused a variation in overlap. For each and every single shot in the photoelectron spectrum, the TEO system provided the relative arrival time of the NIR laser pulse and the electron bunch. In Fig. 2 the measured time of flight spectra are plotted unsorted versus the pulse number as well as sorted according to the corresponding arrival time determined by TEO. The sorting procedure recovers the temporal ordering of the first and second sidebands, visible as light colored patches and labeled SB1 and SB2. From the temporal width of the sideband peaks, one can derive a benchmark number for the accuracy of TEO. The optimal temporal width $\Delta T_{\text {opt }}$ of the sideband is defined by the width of the convolution of both laser pulses as one can assume that the interaction of both pulses with the Xe atom is instantaneous. The width of the NIR laser pulse at the experiment was measured using the well established SHG-FROG technique, to be $51 \mathrm{fs}$ rms. The XUV pulse duration is estimated to be $13 \mathrm{fs}$ $\mathrm{rms}^{1}$ The intrinsic width of sideband appearance is equal to the width of the temporal measured convolution of both pulses. Thus, the shortest possible width $\Delta T_{\text {opt }}$, which could be measured, is calculated from the convolution of both pulses to $53 \mathrm{fs}$ rms. By comparing this optimal width with the width obtained in the experiment $\Delta T_{\mathrm{SB}}=100$ fs rms (Fig. 2 ), one achieves a number for the detection error of TEO

$$
\Delta T_{\mathrm{TEO}, \mathrm{rms}}=\sqrt{\Delta T_{\mathrm{SB}}^{2}-\Delta T_{\mathrm{opt}}^{2}} .
$$

The equation gives rise to $\Delta T_{\mathrm{TEO}, \mathrm{rms}} \approx 87 \mathrm{fs} \mathrm{rms}$. In a second experiment, photoelectron spectra were measured during a delay scan of the NIR laser relative to the FEL over a time range of 2.6 ps. The solid line in Fig. 3 shows the delay time variation of the intensity of the first order sideband as an average over all spectra taken at the respective delay value. The red line was obtained by correcting the delay stage time with the actual jitter time as measured by TEO. The temporal resolution of a pump-probe experiment is defined by the fast-
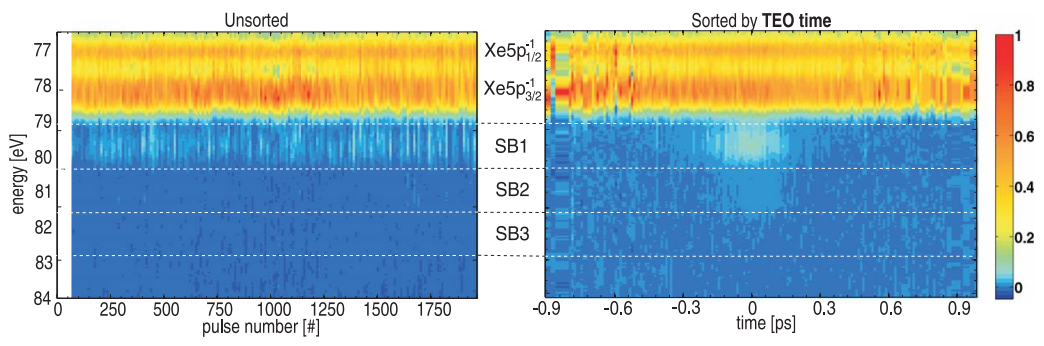

FIG. 2. (Color online) Left: photoelectron spectra plotted as function of the pulse number, Right: spectra sorted according to the arrival time determined by TEO. The spectra are background subtracted and averaged over time intervals of $10 \mathrm{fs}$. The spectral intensity is color encoded. In the arrival time sorted case, two sideband peaks appear. Unsorted no temporal sideband peaks are visible. 


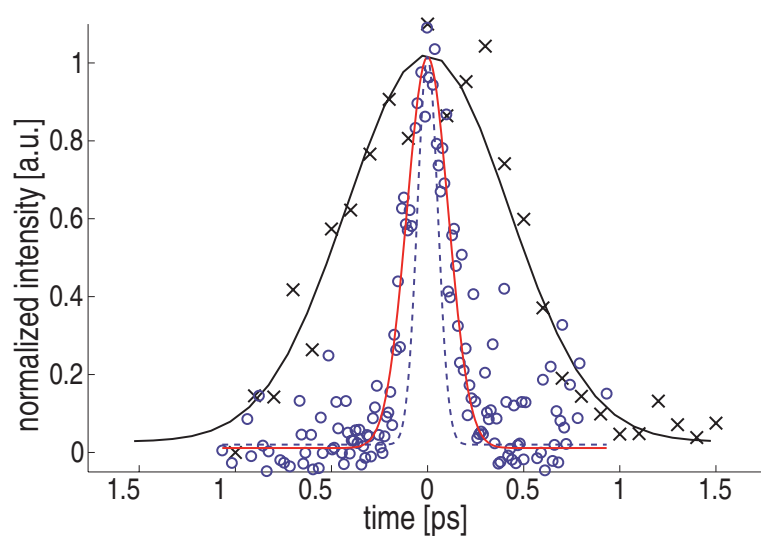

FIG. 3. (Color online) An uncorrected pump-probe delay scan (x) of the normalized amplitude of the first order sideband is compared with the TEO sorted sideband amplitude (o) deduced from Fig. 2 and the expected optimal first order sideband signal (-). A Gaussian peak fit of the uncorrected data shows a width of $410 \mathrm{fs}$ rms. For the corrected sideband peak a width of 100 $\mathrm{fs}$ rms is found. The ideal peak width would be $53 \mathrm{fs}$ rms. Normalized signal amplitudes are used.

est possible temporal structure, which can be resolved temporally. Within this measurement the temporal resolution is increased very significantly from about $400 \mathrm{fs}$ rms (due to the arrival time jitter and drift of FLASH) to $100 \mathrm{fs}$ rms $\left(\Delta T_{\mathrm{SB}}\right)$ with support of the TEO diagnostic. For comparison a Gaussian line with theoretically expected width of $53 \mathrm{fs}$ rms (cross correlation width between FEL and the optical laser) is shown in Fig. 3 as dashed curve. The remaining difference between this optimal curve and the TEO-corrected curve is due to the temporal resolution of TEO. A noninvasive, electro-optical online timing system for support of pump-probe experiments at FLASH has been set up. A benchmarking pump-probe experiment has shown that the timing system has an accuracy better than $90 \mathrm{fs}$ rms $\left(\Delta T_{\mathrm{TEO}}\right)$. The diagnostic is ready for user operation and becomes standard diagnostic for pump-probe experiments with demands for high temporal resolution at the FLASH facility.

The support of the FLASH user facility, in particular funding of the laser system through the BMBF program FSP301-FLASH is greatly acknowledged.

${ }^{1}$ W. Ackermann et al., Nat. Photonics 1, 336 (2007).

${ }^{2}$ M. Meyer, D. Cubaynes, D. Glijer, J. Dardis, P. Hayden, P. Hough, V. Richardson, E. T. Kennedy, J. T. Costello, P. Radcliffe, S. Düsterer, A Azima, W. B. Li, H. Redlin, J. Feldhaus, R. Taïeb, A. Maquet, A. N. Grum-Grzhimailo, E. V. Gryzlova, and S. I. Strakhova, Phys. Rev. Lett. 101, 193002 (2008).

${ }^{3}$ P. Radcliffe, S. Düsterer, A. Azima, H. Redlin, J. Feldhaus, J. Dardis, K. Kavanagh, H. Luna, J. Pedregosa Gutierrez, P. Yeates, E. T. Kennedy, J. T. Costello, A. Delserieys, C. L. S. Lewis, R. Taïeb, A. Maquet, D. Cubaynes, and M. Meyer, Appl. Phys. Lett. 90, 131108 (2007).

${ }^{4}$ M. Meyer, D. Cubaynes, P. O’Keeffe, H. Luna, P. Yeates, E. T. Kennedy, J. T. Costello, P. Orr, R. Taïeb, A. Maquet, S. Düsterer, P. Radcliffe, H. Redlin, A. Azima, E. Plönjes, J. Feldhaus, Phys. Rev. A. 74, 011401(R) (2006).

${ }^{5}$ C. Gahl, A. Azima, M. Beye, M. Deppe, K. Döbrich, U. Hasslinger, F. Hennies, A. Melnikov, M. Nagasono, A. Pietzsch, M. Wolf, W. Wurth, and A. Föhlisch, Nat. Photonics 2, 165 (2008).

${ }^{6}$ A. Barty, S. Boutet, M. J. Bogan, S. Hau-Riege, S. Marchesini, K. Sokolowski-Tinten, N. Stojanovic, R. Tobey, H. Ehrke, A. Cavalleri, S. Düsterer, M. Frank, S.Bajt, B. W. Woods, M. M. Seibert, J. Hajdu, R. Treusch, and H. N. Chapman, Nat. Photonics 2, 415 (2008).

${ }^{7}$ T. Maltezopoulos, S. Cunovic, M. Wieland, M. Beye, A. Azima, H. Redlin, M. Krikunova, R. Kalms, U. Frühling, F. Budzyn, W. Wurth, A. Föhlisch, and M. Drescher, New J. Phys. 10, 10 (2008).

${ }^{8}$ A. Cavalieri et al., Phys. Rev. Lett. 94, 114801 (2005).

${ }^{9}$ J. Kim, J. Chen, Z. Zhang, F. N. C. Wong, F. X. Kärtner, F. Loehl, and H. Schlarb, Opt. Lett. 32, 9 (2007).

${ }^{10}$ A. Azima, S. Düsterer, H. Schlarb, J. Feldhaus, A. Cavalieri, D. Fritz, and K. Sengstock, Proceedings of the EPAC, Edinburgh, 2006 (unpublished). 